\title{
Clinical Features of Fracture versus Concussion of the Temporal Bone after Head Trauma
}

\author{
Tae Hoon Kong, Jae Woo Lee, Yoon Ah Park, and Young Joon Seo \\ Department of Otorhinolaryngology-Head and Neck Surgery, Yonsei University Wonju College of Medicine, Wonju, Korea
}

Received July 2, 2018

Revised August 23, 2018

Accepted September 4, 2018
Background and Objectives: Temporal bone fracture (TBF) is a common occurrence in cases of head trauma. Although the incidence of temporal bone concussion (TBC) has increased in cases of head trauma, it has not been extensively studied. We assessed the characteristics of TBF and TBC in patients with head trauma. Subjects and Methods: We conducted a retrospective review of 432 patients with head injury who visited our hospital between January 2011 and April 2016. Of these patients, 211 who met the inclusion criteria were included in the study. Their clinical characteristics, causes of injury, and hearing function were analyzed. Results: Among the 211 patients, 157 had TBFs and 54 had TBCs. Ear symptoms were more common among patients with TBF than among those with TBC. Car accidents were the most common cause of both TBF and TBC, but assault and sports injuries were more common among patients with TBC than among those with TBF. The occurrence of facial palsy in both cases of TBF and TBC. Hearing loss was observed among 35 patients with TBF and 11 patients with TBC. However, patients with TBF showed conductive hearing loss with an air-bone gap. Hearing function of these patients with TBF recovered with a reduced air-bone gap, but the patients with TBC showed little recovery. Conclusions: Emergency physicians should focus more on temporal bone injury in patients with head trauma. Therefore, an early complete diagnostic battery, which includes high-resolution computed tomography, audiometric tests, neurologic examination, and vestibular tests, be performed in patients with head trauma.

J Audiol Otol 2019;23(2):96-102

\section{Introduction}

Temporal bone fracture (TBF) has been reported to occur in about $30 \%$ to $70 \%$ of cases of skull fractures in patients with head trauma [1]. TBFs have been extensively studied because of their severity and emergency nature [2-4]. Otologic symptoms, including ear bleeding, facial palsy, and dizziness, generally make physicians suspect temporal bone injury.

Conventionally, TBFs are divided into the transverse, longitudinal, and mixed types depending on the direction of the fracture line [5]. Many recent studies have suggested that TBFs should be categorized into the otic-capsule-violating and otic-

This is an Open Access article distributed under the terms of the Creative Commons Attribution Non-Commercial License (https://creativecommons.org/licenses/by-nc/4.0/) which permits unrestricted non-commercial use, distribution, and reproduction in any medium, provided the original work is properly cited. capsule-sparing types, or on the basis of whether the involvement of the petrous bone is correlated with the severity of symptoms and clinical relevance [6,7]. The patients' symptom progression and prognosis vary according to the type of fracture. Therefore, determining whether a patient has a TBF and the type of TBF is important in the emergency room. However, injury to the temporal bone is often overlooked during the initial evaluation and management of patients presenting to the emergency department with severe head trauma [3].

The incidence of temporal bone concussion (TBC), also called labyrinthine concussion, has increased in cases of head trauma, but it has not been extensively studied [8,9]. Many patients with TBC also present otologic symptoms, such as hearing loss, tinnitus, dizziness, and headache, but the symptoms do not improve easily even after several years [9-11].

In this study, we assessed the characteristics of TBF and 
TBC in patients with head trauma, and utilized the characteristics to predict and explain the prognosis to the patients. Our study findings could provide evidence supporting the development of guidelines to ensure patients receive proper management after the initial evaluation.

\section{Subjects and Methods}

\section{Subjects}

We performed a retrospective study on patients with head injury, irrespective of whether they had other lesions or injuries, who visited the emergency trauma center of Yonsei University Wonju Severance Christian Hospital between January 2011 and April 2016.

The exclusion criteria were as follows: 1) patients who were dead on arrival; 2) patients who had been transferred to other hospitals upon their request; 3) patients with a known previous history of chronic otitis media or hearing loss; 4) patients without otologic symptoms or who had not undergone otologic evaluations; and 5) patients who had not undergone high-resolution temporal bone computed tomography (TBCT). However, if the patients had undergone brain computed tomography (CT) that revealed a TBF, then the patients were included our study. Finally, 211 patients were enrolled in our study (Fig. 1).

Data on clinical characteristics such as age, sex, injury site (right or left), and ear symptoms were collected. The cause of head injury, mean threshold of pure tone audiometry (PTA), and the PTA value of frequencies ranging from $250 \mathrm{~Hz}$ to 4,000 Hz were collected.

\section{TBC vs. TBF}

We defined and categorized TBFs according to the conventional method following the direction of the fracture line in reference to the petrous ridge into longitudinal, transverse, oblique, mixed, or comminuted fractures. We defined TBC according to the following criteria: 1) history of head trauma without any abnormal findings on radiologic studies; 2) inner ear symptoms such as hearing disturbance, dizziness and tinnitus after trauma; 3) no history of hearing disturbance before trauma; and 4) if the hearing disturbance was presented, sensorineural hearing loss on PTA greater than $25 \mathrm{~dB}$ according to the American Academy of Otolaryngology-Head and Neck Surgery standard method. Patients with a history of previous hearing loss or chronic otitis media and showing signs of conductive hearing loss or perilymphatic fistula were excluded.

\section{Statistical analysis}

Statistical analysis was performed using IBM SPSS Statistics for Windows (Version 22.0, IBM Corp., Armonk, NY, USA). We used Fisher's exact test and chi-square test for categorized variables, and independent t-test and paired t-test for continuous variables. A $p$-value less than $0.05(p<0.05)$ was considered to indicate a significant difference.

\section{Results}

\section{Clinical features of TBF and TBC}

The age of the patients ranged from 7 to 91 years, and their mean age was 47.53 years. The mean ages were similar for the TBC and TBF groups (45.24 and 48.32 years, respectively, $p$-value $=0.345)$. Both injury types were more frequent among
Fig. 1. Flow diagram illustrating the selection of subjects with TBF and TBC. ER: emergency room, COM: chronic otitis media, TBCT: temporal bone computed tomography, TBC: temporal bone concussion, TBF: temporal bone fracture.

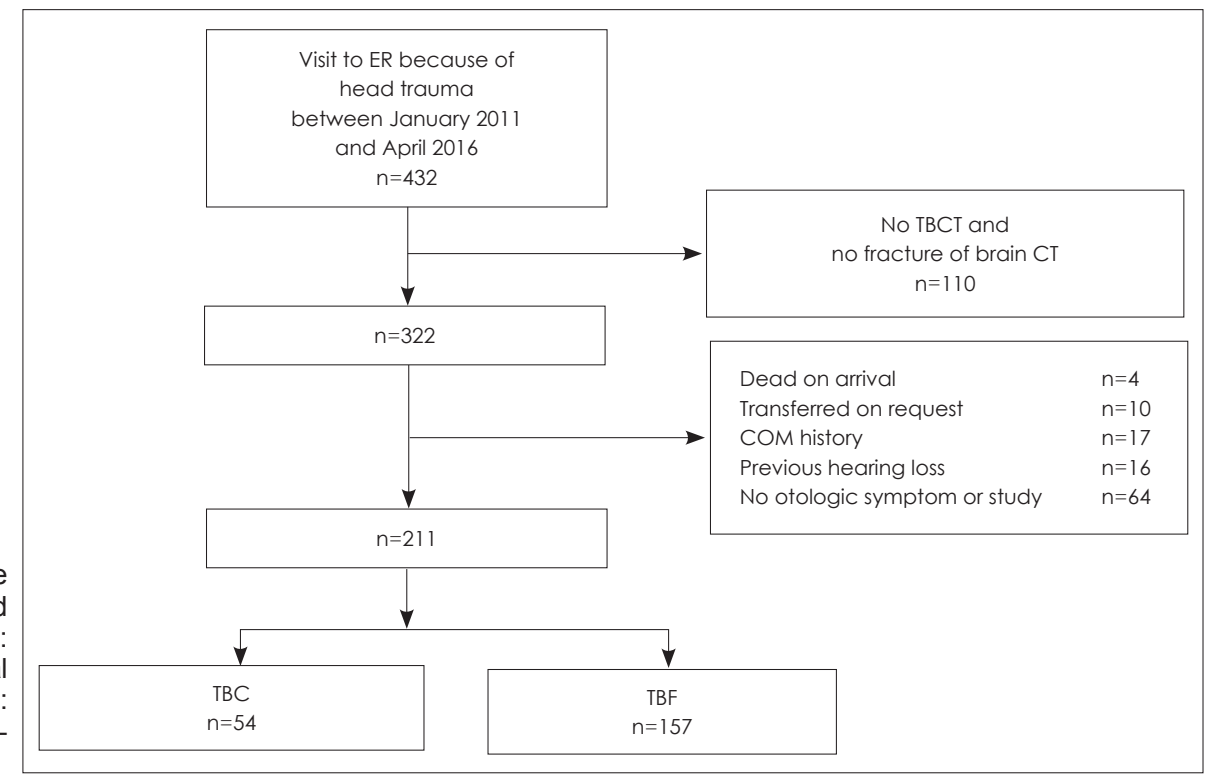


Table 1. Clinical features of TBC and TBF

\begin{tabular}{lccc}
\hline Clinical feature & TBC $(n=54)$ & TBF $(n=157)$ & $p$-value \\
\hline Age (years) & $45.24 \pm 20.39$ & $48.32 \pm 20.98$ & 0.345 \\
Sex (Male) & $44(81.5)$ & $123(78.3)$ & 0.701 \\
Site & & & $<0.001^{*^{\dagger}}$ \\
$\quad$ Right & $15(27.8)$ & $78(49.7)$ & \\
Left & $23(42.6)$ & $69(43.9)$ & \\
Both & $5(9.3)$ & $10(6.4)$ & \\
Unknown & $11(20.4)$ & $0(0.0)$ & \\
Ear symptoms & & & \\
Otorrhea & $12(23.1)$ & $106(68.4)$ & $<0.001^{*}$ \\
Hemotympanum & $5(9.6)$ & $99(64.3)$ & $<0.001^{*}$ \\
EAC stenosis & $0(0.0)$ & $23(16.3)$ & $0.002^{*}$ \\
Drum perforation & $2(4.0)$ & $21(15.9)$ & $<0.050^{*}$ \\
CSF leakage & $0(0)$ & $5(3.2)$ & 0.184 \\
Comorbidity & & & \\
CVA & $31(57.4)$ & $119(77.3)$ & $0.008^{*}$ \\
Skull fracture & $17(31.5)$ & $102(66.2)$ & $<0.001^{*}$ \\
Spine fracture & $6(11.1)$ & $15(9.7)$ & 0.795 \\
Facial fracture & $11(20.4)$ & $37(24.0)$ & 0.708 \\
\hline Valves are exprear & & & \\
\hline
\end{tabular}

Values are expressed as mean $\pm S D$, or as number (percentage). *p-value<0.05, 'Fisher's exact test. EAC: external auditory canal, CSF: cerebrospinal fluid, CVA: cerebrovascular accident, TBC: temporal bone concussion, TBF: temporal bone fracture, SD: standard deviation

male subjects than among female subjects (Table 1).

In the TBF group, the incidence of injury was similar on the right and left sides, but in the TBC group, injuries were more frequent on the left side than on the right side. Of the 54 patients with TBC, 11 (20.4\%) suffered only dizziness, which prevented the determination of the lesion side (Table 1).

Ear symptoms such as bloody otorrhea, hemotympanum, external auditory canal stenosis, tympanic membrane perforation, and cerebrospinal fluid (CSF) leakage were more common in the TBF group than in the TBC group, and the intergroup differences were statistically significant, except for CSF leakage (Table 1).

The comorbidities associated with injury, such as cerebrovascular accident (CVA) and skull fracture, were more frequent in the TBF group than in the TBC group, but the incidence of spine fracture and facial bone fracture were similar between the two groups (Table 1).

Table 2 shows the causes of injuries. We categorized the causes into six groups: car accident, fall, assault, industrial injury, sports injury, and others. Car accident was the most common cause of injury in both groups, and was particularly more common in the TBF group than in the TBC group. The proportion of fall injuries was similar in the TBC and TBF groups (27.8\% and 28.7\%, respectively). Most patients with assault and sports injury were in the TBC group, except for one pa-
Table 2. Cause of injury in cases of TBC and TBF

\begin{tabular}{lccc}
\hline \multicolumn{1}{c}{ Cause } & TBC $(n=54)$ & TBF $(n=157)$ & p-value \\
\hline Car accident & $19(35.2)$ & $91(58.0)$ & $<0.001^{* \dagger}$ \\
Fall & $15(27.8)$ & $45(28.7)$ & \\
Assault & $6(11.1)$ & $1(0.6)$ & \\
Industrial injury & $5(9.3)$ & $9(5.7)$ & \\
Sports injury & $3(5.6)$ & $1(0.6)$ & \\
Others & $6(11.1)$ & $10(6.4)$ &
\end{tabular}

Values are expressed as number (percentage). *p-value<0.05, ${ }^{\dagger}$ Fisher's exact test. TBC: temporal bone concussion, TBF: temporal bone fracture

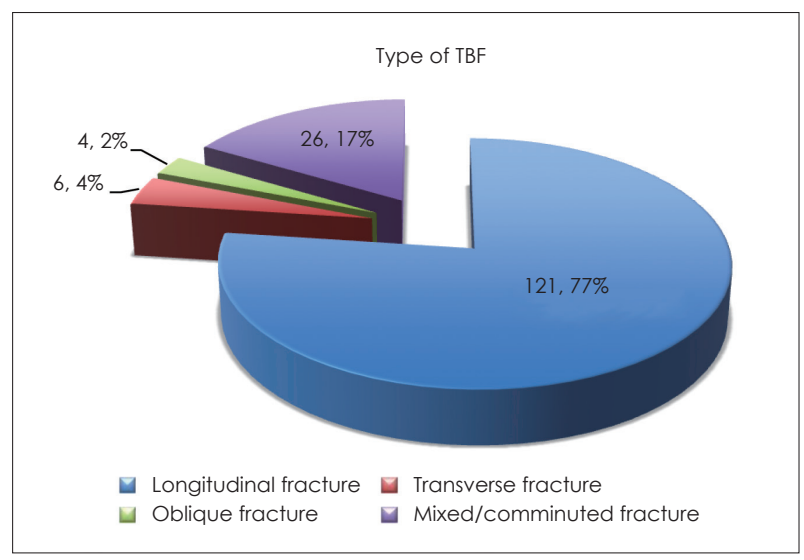

Fig. 2. Type of TBF. TBF: temporal bone fracture.

tient each in the TBF group (Table 2).

In the TBF group, longitudinal fracture was the most common type (77\%), followed by mixed/comminuted (17\%), transverse (4\%), and oblique (2\%) fractures (Fig. 2).

Facial palsy occurred only in the TBF group, with an incidence of $18.3 \%$ (Table 3 ). The average value of electroneuronography performed between 3 and 20 days after facial palsy was $63.71 \%$. The prognosis of facial palsy is shown in Fig. 3. The mean initial House-Brackmann grade (H-B grade) was 2.82, and the patients recovered to a $\mathrm{H}-\mathrm{B}$ grade of 1.56. The follow-up period for patients with facial palsy was ranged 7 to 46 days, and 24.67 days of mean.

\section{Hearing function changes after TBF and TBC}

We analyzed hearing progression among the patients. To observe the trends in hearing changes after trauma, we excluded 43 patients from the TBC group and 122 from the TBF group who had not undergone PTA or had undergone PTA just once, which prevented the observation of the trends in hearing changes. Finally, 11 of the 54 patients with TBC and 35 of the 157 with TBF were analyzed. The mean interval between injury and the initial PTA in both groups was 16.96 days. In the TBF group, the mean interval between injury and the initial PTA was 18.14 days, and in the TBC group the interval 
Table 3. Prognosis of facial palsy in TBF

\begin{tabular}{lccc}
\hline & Concussion & TBF & p-value \\
\hline Facial palsy & $0(0.0)$ & $24(18.3)$ & $0.001^{*}$ \\
Initial H-B grade & & $2.82 \pm 1.01$ & \\
ENoG (\%) & & $63.71 \pm 17.56$ & \\
Interval (days) & $24.67 \pm 17.54$ & \\
Final H-B grade & & $1.56 \pm 0.89$ & \\
\hline
\end{tabular}

Values are expressed as mean $\pm S D$, or as number (percentage). *p-value<0.05. H-B grade: House-Brackmann grade, ENoG: electroneuronography, TBF: temporal bone fracture, SD: standard deviation

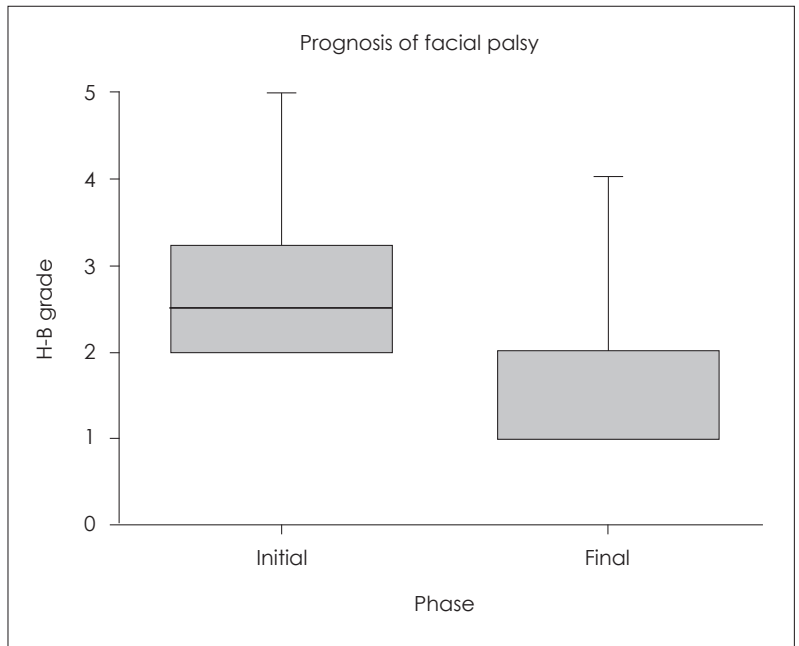

Fig. 3. Prognosis of facial palsy in patients with TBF. H-B grade: House-Brackmann grade. TBF: temporal bone fracture.

was 13.93 days, but no statistically significant intergroup difference was observed ( $p$-value $=0.207)$.

Fig. 4 shows the mean hearing ability in both groups. The TBC group showed sensorineural hearing loss without conductive hearing loss and a mean PTA threshold of $57.77 \mathrm{~dB}$ HL. In contrast, the TBF group showed conductive hearing loss with a mean PTA threshold of $51.89 \mathrm{~dB}$ HL for air conduction and $24.61 \mathrm{~dB}$ HL for bone conduction. In both groups, hearing impairment was more severe at high frequencies.

We also tracked the prognosis of hearing function. Patients were followed up for a mean duration of 110.21 days (range, 8 to 382 days) in the TBF group and 69.80 days (range, 3 to 331 days) in the TBC group ( $p$-value $=0.317$ ). Proper management such as explore-tympanotomy with or without tympanoplasty for conductive hearing loss, or empirical high dose steroid therapy for sensorineural hearing loss resulted in a mild improvement in hearing ability on air conduction in the TBF group with a mean PTA value of $10.47 \mathrm{~dB}$ ( $p$-value= $0.002)$. However, an exacerbation of hearing loss on bone conduction was observed with a mean PTA value of $2.91 \mathrm{~dB}$.

In the TBC group, the patients showed only slight changes in hearing ability on air conduction. The mean PTA value was

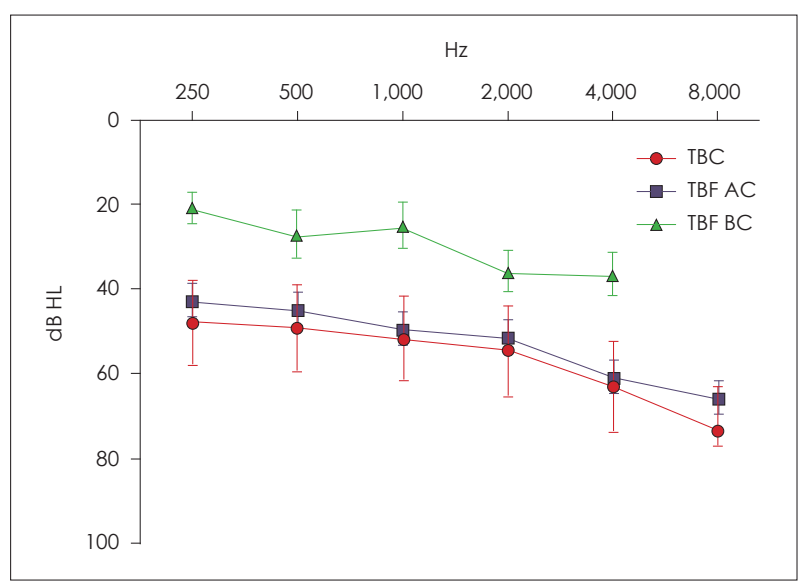

Fig. 4. Initial hearing level of in the TBF and TBC groups. TBC: temporal bone concussion, TBF: temporal bone fracture, AC: air conduction, BC: bone conduction.

slightly improved at $2.5 \mathrm{~dB}$ ( $p$-value $=0.685)$, but the hearing improvement varied according to the frequencies. Moreover, as the frequencies increased, the hearing ability worsened (Fig. 5).

With respect to the PTA frequencies, in the TBF group, the mean improvement in the PTA value of air conduction was $6.62 \mathrm{~dB} \mathrm{HL}$ at $250 \mathrm{~Hz}, 9.14 \mathrm{~dB} \mathrm{HL}$ at $500 \mathrm{~Hz}, 10.43 \mathrm{~dB} \mathrm{HL}$ at $1,000 \mathrm{~Hz}, 10.57 \mathrm{~dB} \mathrm{HL}$ at $2,000 \mathrm{~Hz}, 9.14 \mathrm{~dB} \mathrm{HL}$ at 4,000 $\mathrm{Hz}$, and $9.39 \mathrm{~dB} \mathrm{HL}$ at $8,000 \mathrm{~Hz}$. All differences were statistically significant except at $250 \mathrm{~Hz}$ (the corresponding $p$-values were $0.093,0.033,0.009,0.001,0.005,0.008$, and 0.002, respectively). In the $\mathrm{TBC}$ group, the mean improvement in PTA value was $4.09 \mathrm{~dB}$ at $250 \mathrm{~Hz}, 4.55 \mathrm{~dB}$ at $500 \mathrm{~Hz}, 0.00$ $\mathrm{dB}$ at $1,000 \mathrm{~Hz}$, and $4.09 \mathrm{~dB}$ at $2,000 \mathrm{~Hz}$, and exacerbation of hearing loss was $0.91 \mathrm{~dB}$ at $4,000 \mathrm{~Hz}$ and $2.27 \mathrm{~dB}$ at 8,000 $\mathrm{Hz}$, but none of the differences were statistically significant.

\section{Discussion}

The regional emergency medical center and trauma center of our institute is the sole tertiary hospital in our state, and three highways pass near our city. Moreover, our city is a gateway between the crowded capital territory and a state famous among tourists. Because of the geographical characteristics of our hospital, many patients with severe trauma are transferred to our hospital, and in many cases of trauma due to car accidents, the patients' homes are far from our city. Therefore, after the management of acute, critical, and urgent injuries, many patients are referred to other hospitals near their home upon request.

Among the causes of injury, car accident was more common in the TBF group than in the TBC group, even though car accident was the most common cause of both injuries. The in- 

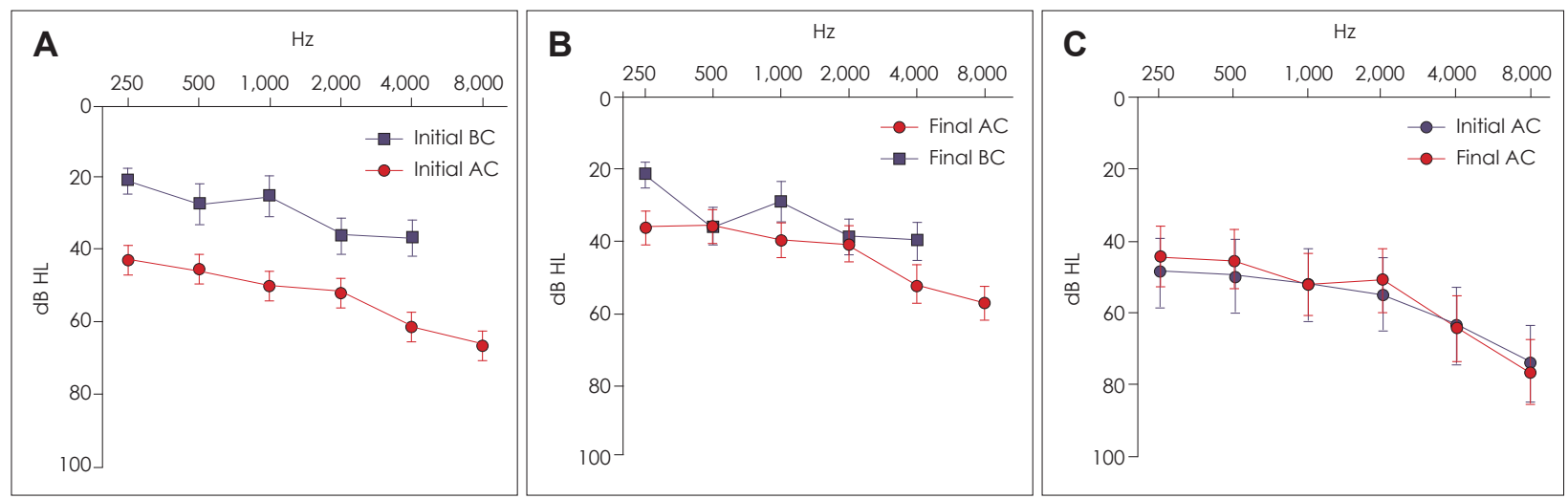

Fig. 5. Prognosis of hearing level in the TBF and TBC groups. A: Mean initial hearing level in the TBF group. B: Mean final hearing level in the TBF group. C: Mean initial and final hearing levels in the TBC group. TBF: temporal bone fracture, TBC: temporal bone concussion, BC: bone conduction, AC: air conduction.

cidence of TBC caused by car accident (58.0\%) in this study was very similar to that in a study conducted by the University of California that analyzed 820 TBFs (57.79\%) [2]. However, although injuries caused by car accidents were the most common in the TBC group, the incidence (35.2\%) was lower than that in the TBF group. In contrast, Choi, et al. [11] reported a significant contribution of traffic accidents $(87.5 \%)$ to the incidence of labyrinthine concussion. As described above, three highways pass near our city, and the high number of car accidents contributes to severe head trauma, which may contribute to the high incidence of TBFs, rather than TBCs. In our study, among the 211 patients with head traumas, 110 were involved in car accidents, and 91 of them had TBFs ( $82.73 \%$ ).

Fall injuries had a similar distribution between the TBC and TBF groups (27.8\% and $28.7 \%$, respectively). However, the incidence of assault and sports injury was significantly higher in the TBC group than in the TBF group. Among seven patients who experienced assault and four with sports injury resulting in head trauma, only one patient each had a TBF.

Trauma caused by assault usually occurs on the left side, because most attackers are right-handed [12]. Our findings showed that unlike in the TBF group, the TBC group had more frequent injuries on the left side than on the right side. The higher incidence of assaults in the TBC group contributes to the higher incidence of left-sided injuries.

On the basis of our results, we presume that when a patient experiences head trauma because of a car accident, TBF is more likely to occur than is TBC. We also presume that most of the patients with head trauma caused by assault and sports injury will have TBCs. No incidences of gunshot injury were noted in our study, unlike in the study by the University of California, because the possession of firearms by ordinary people is illegal in South Korea [2].

In our study, facial palsy occurred only in the TBF group; 24 of the 157 (18.3\%) patients with TBF experienced facial palsy. Other reports have documented 56 of 820 patients $(6.83 \%)$ and 4 of 61 patients $(6.56 \%)$ developing facial nerve paralysis after head trauma with TBF [2,3]. Sanus, et al. [13] reported 25 cases of delayed facial nerve paralysis without TBF. They did not report the incidence of facial nerve paralysis in patients with TBC, but they reported that facial palsy can occur with H-B grades ranging from 3 to 6 , which were treated medically and even surgically [13]. Another study reported that facial nerve palsy occurred in 40 of 794 patients $(5.04 \%)$ after head trauma [14]. The incidence of facial nerve palsy is mildly higher in our study than in other reports. Regardless of whether a patient has a TBF or TBC, we should monitor the development of facial palsy after head trauma. However, because of the short follow-up period, the final outcome of facial palsy was not presented in our study.

In case of hearing impairment, both the TBC and TBF groups showed similar initial air conduction on PTA (57.77 $\mathrm{dB}$ and $51.89 \mathrm{~dB}$ ). Most patients with TBF and hearing impairment experienced conductive hearing loss. In contrast, patients with TBC and hearing impairment experienced sensorineural hearing loss. Conductive hearing loss is due to middle ear injuries, such as hemotympanum and ossicular disruption. Kim, et al. [15] reported larger air-bone gaps (AB gaps) in patients with ossicular chain involvement than in those with no ossicular chain involvement, which is caused by hematoma and hemotympanum. Grant, et al. [16] reported that $\mathrm{AB}$ gaps decreased from $24.8 \mathrm{~dB}$ to $13.2 \mathrm{~dB}$ on average. In our TBF group, $A B$ gaps decreased from an average of $27.28 \mathrm{~dB}$ to $13.90 \mathrm{~dB}$. Considering the $10.47 \mathrm{~dB}$ mean improvement in air conduction and $2.91 \mathrm{~dB}$ mean exacerbation in bone conduction, we can suggest that as a comprehensive approach, the $\mathrm{AB}$ gap in conductive hearing loss in the TBF group decreased because of the dual action of an improvement in air conduction and an exacerbation of bone conduction. However, hearing loss after TBF does not always recover. 
When the pneumolabyrinth is concomitant with TBF, hearing loss may progress to near deafness and may not improve [17].

The average value of hearing impairment among patients with TBC showed little improvement. In particular, at high frequencies, hearing function worsens over time. Choi, et al. [11] reported that hearing function at high frequencies was more vulnerable to trauma than was that at low frequencies in patients with labyrinthine concussion. They showed that only 13 of their 40 patients partially recovered their hearing impairment, while the other 27 showed no change or worse hearing ability [11]. Ulug and Ulubil [9] reported 3 cases of patients with labyrinthine concussion; they also showed most severe hearing impairment at a frequency of $4,000 \mathrm{~Hz}$, and did not recover hearing function after a 1-year follow-up. The high-frequency hearing loss caused by cochlear concussion in both $\mathrm{TBF}$ and $\mathrm{TBC}$ is because of basilar membrane shearing, hair cell degeneration, and eventual auditory nerve fiber avulsion [18]. Therefore, because head trauma can have a negative effect on hearing function and can worsen sensorineural hearing loss in both TBC and TBF, early evaluation and intervention are necessary to prevent hearing loss.

\section{Limitation}

In our study, all patients with head trauma did not undergo PTA, and the initial hearing evaluation was performed later in the TBF group than in the TBC group. In severe cases of head trauma, the patients may develop serious comorbidities, such as CVA, which might lead to sensorineural hearing loss or affect the patients' general condition preventing them from undergoing PTA in an audio booth. This bias could have contributed to the better mean PTA threshold in the TBF group than in the TBC group.

Many cases of TBF are accompanied by severe comorbidities, such as cerebral hemorrhage, and because of its emergency nature, many emergency physicians could overlook the evaluation of temporal bone injury, especially in cases in which the patient has few symptoms. Although brain CT is frequently performed in most cases of head injury to assess cerebral hemorrhage, high-resolution TBCT is less frequently performed in such cases at our emergency center. In our study, among 432 patients with head trauma, 124 (28.70\%) did not undergo high-resolution TBCT. Consequently, in many cases, high-resolution TBCT was performed only when the patients showed otologic symptoms, such as hearing loss, tinnitus, or dizziness. Moreover, there is a possibility that high-resolution TBCT cannot distinguish between TBC and spontaneous fusion in TBF. Therefore, we suggest that head trauma requires a complete diagnostic battery, which includes a neurologic examination, high-resolution TBCT, audiometric tests, and vestibu- lar tests if required [19]. If an emergency physician cannot order some of these evaluations because of the patient's mental status or the need for emergency operation, the patient should undergo these tests immediately after the emergency has been addressed.

The data on tinnitus and dizziness were not analyzed in our study because of the small number of cases and the high number of patients lost to follow-up. Our study cannot provide the clinical characteristics of dizziness and tinnitus in patients with temporal bone injury. Further studies should be performed to reveal their characteristics and prognosis.

In this study, we divided the patients into two groups on a case-by-case basis, but we did not consider the possibility that some patients could have TBF on one side and TBC on the other side $[9,19]$. Future studies should consider this possibility of TBC and TBF occurring together in patients.

\section{Conclusion}

Car accident was the most common cause of head trauma in both the TBF and TBC groups. However, assault and sports injury were more common in the TBC group than in the TBF group. Although no facial nerve palsy was observed among patients with TBC, considering the findings of other studies, delayed facial nerve palsy may occur in patients with TBC. Therefore, we should monitor the development of facial nerve palsy in all patients with head trauma. Hearing loss in TBF is characterized by AB gaps, which decrease with hearing improvement. Moreover, sensorineural hearing loss was exacerbated in TBF, as well as in TBC, especially at high frequencies. Emergency physicians should focus more on temporal bone injury in patients with head trauma. Therefore, we suggest that patients with head trauma should undergo an early complete diagnostic battery performed by an otolaryngologist, and the battery should include a high-resolution TBCT scan, audiometric tests, a neurologic examination, and vestibular tests if required.

\section{Acknowledgments}

This work was supported by the Gangwon Institute for Regional Program Evaluation grant funded by the Korea government (Ministry of Trade, Industry and Energy) (No. R0005797) and by the Technology Innovation Program (or Industrial Strategic Technology Development Program) (20001819) funded By the Ministry of Trade, Industry \& Energy (MOTIE, Korea).

\section{Conflicts of interest}

The authors have no financial conflicts of interest.

\section{REFERENCES}

1) Hasso AN, Ledington JA. Traumatic injuries of the temporal bone. Otolaryngol Clin North Am 1988;21:295-316. 
2) Brodie HA, Thompson TC. Management of complications from 820 temporal bone fractures. Am J Otol 1997;18:188-97.

3) Amin Z, Sayuti R, Kahairi A, Islah W, Ahmad R. Head injury with temporal bone fracture: one year review of case incidence, causes, clinical features and outcome. Med J Malaysia 2008;63:373-6.

4) Nicol JW, Johnstone AJ. Temporal bone fractures in children: a review of 34 cases. J Accid Emerg Med 1994;11:218-22.

5) Ishman SL, Friedland DR. Temporal bone fractures: traditional classification and clinical relevance. Laryngoscope 2004;114:1734-41.

6) Dahiya R, Keller JD, Litofsky NS, Bankey PE, Bonassar LJ, Megerian CA. Temporal bone fractures: otic capsule sparing versus otic capsule violating clinical and radiographic considerations. J Trauma 1999; 47:1079-83.

7) Kang HM, Kim MG, Boo SH, Kim KH, Yeo EK, Lee SK, et al. Comparison of the clinical relevance of traditional and new classification systems of temporal bone fractures. Eur Arch Otorhinolaryngol 2012;269:1893-9.

8) Gaw CE, Zonfrillo MR. Emergency department visits for head trauma in the United States. BMC Emerg Med 2016;16:5.

9) Ulug T, Ulubil SA. Contralateral labyrinthine concussion in temporal bone fractures. J Otolaryngol 2006;35:380-3.

10) Chiaramonte R, Bonfiglio M, D'Amore A, Viglianesi A, Cavallaro $\mathrm{T}$, Chiaramonte I. Traumatic labyrinthine concussion in a patient with sensorineural hearing loss. Neuroradiol J 2013;26:52-5.

11) Choi MS, Shin SO, Yeon JY, Choi YS, Kim J, Park SK. Clinical characteristics of labyrinthine concussion. Korean J Audiol 2013;17:
13-7.

12) Hitchin AD, Shuker ST. Some observations on zygomatic fractures in the Eastern Region of Scotland. Br J Oral Surg 1973;11:114-7.

13) Sanus GZ, Tanriverdi T, Tanriover N, Ulu MO, Uzan M. Hearing preserved traumatic delayed facial nerve paralysis without temporal bone fracture: neurosurgical perspective and experience in the management of 25 cases. Surg Neurol 2009;71:304-10, discussion 310.

14) Odebode TO, Ologe FE. Facial nerve palsy after head injury: case incidence, causes, clinical profile and outcome. J Trauma 2006;61: 388-91.

15) Kim SY, Kim YJ, Kim YH, Park MH. Audiologic patterns of otic capsule preserving temporal bone fracture: effects of the affected subsites. Clin Exp Otorhinolaryngol 2016;9:206-11.

16) Grant JR, Arganbright J, Friedland DR. Outcomes for conservative management of traumatic conductive hearing loss. Otol Neurotol 2008;29:344-9.

17) Choi HG, Lee HJ, Lee JS, Kim DH, Hong SK, Park B, et al. The rates and clinical characteristics of pneumolabyrinth in temporal bone fracture. Otol Neurotol 2015;36:1048-53.

18) Valvassori GE. Imaging of the temporal bone. In: Snow JB, Ballenger JJ, editors. Ballenger's otorhinolaryngology: head and neck surgery. Baltimore: Williams \& Wilkins;2003. p.195-229.

19) Villarreal IM, Méndez D, Silva JM, Del Álamo PO. Contralateral cochlear labyrinthine concussion without temporal bone fracture: unusual posttraumatic consequence. Case Rep Otolaryngol 2016; 2016:2123182. 